\title{
Incipient Motion of Sediments in Oscillatory Flows of Pseudoplastic Fluids: Preliminary Results
}

\author{
Aldo Tamburrino ${ }^{1}$, Kevin Vidal $^{2}$ \\ ${ }^{1}$ Department of Civil Engineering \& Advanced Mining Technology Center, University of Chile \\ Av. Blanco Encalada 2002, Santiago 8370449, Chile \\ atamburr@ing.uchile.cl \\ ${ }^{2}$ Department of Civil Engineering, University of Chile \\ Av. Blanco Encalada 2002, Santiago 8370449, Chile \\ kevin.vidal@ing.uchile.cl
}

\begin{abstract}
Preliminary results of an experimental study on the incipient motion of granular, non-cohesive particles in a laminar oscillatory flow of water and power law fluids are presented in this paper. The threshold condition is defined in terms of two dimensionless variables, one $(\Psi)$ representing the ratio between Coulombic and drag forces acting on the particles, and another $\left(X_{n}\right)$ that involves the quotient of the particle diameter and a scale of the boundary layer thickness, a modified Reynolds number of the particle, and a function of the flow behaviour index of the fluid. A relation between $\Psi$ and $X_{n}$ was obtained that shows good agreement with the experimental data.
\end{abstract}

Keywords: incipient motion, oscillatory flows, power-law fluid, laminar flow

\section{Introduction and Objective of the Paper}

The threshold condition of non-cohesive granular sediment in oscillating flows has been the subject of extensive research when the fluid media is water. Since the classical paper by Bagnold [1], a myriad of research has been published [2-8]. Depending of the author, the threshold condition is expressed in terms of some properties of the fluid, the sediment, and the flow near the bed. The variables used to characterize the incipient motion of the sediment differ according to the researcher, but usually they include variables associated to the orbital motion of the fluid near the bed, such as its period (or frequency) and maximum orbital excursion of the fluid particles. The sediment is characterized by its size and density. The fluid is defined by its density and viscosity. Some authors describe the threshold condition in terms of a shear stress acting on the particle, which frequently is associated to the flow velocity by means of a friction factor.

As far as the authors are aware, all the existing relationships to determine the threshold condition in oscillating flows have been derived considering a Newtonian fluid, namely water, and there is not reported research when the fluid is nonNewtonian. Published studies on the generation of sheet flows, in which the sheet of mud is modeled as a non-Newtonian fluid, deal with a different problem $[9,10]$.

The objective of this paper is to present some results of an ongoing experimental research on the condition for incipient motion of granular non-cohesive sediment under the action of waves of a power law fluid.

\section{Experimental set-up, materials and methods}

\subsection{Experimental set-up}

A sketch of the experimental set-up used in the research is shown in Fig. 1. It consists in a tank $4 \mathrm{~m}$ long, $0.15 \mathrm{~m}$ wide and $0.4 \mathrm{~m}$ high, with a sand bed $2.15 \mathrm{~m}$ long and $4 \mathrm{~cm}$ thick. The tank is filled with fluid up to a defined height and waves are generated by a paddle moved by a $1 \mathrm{HP}$ motor. A gear mechanism and an electronic frequency regulator control the motion of the paddle. The stroke of the paddle can be adjusted between 0 and $8 \mathrm{~cm}$. 


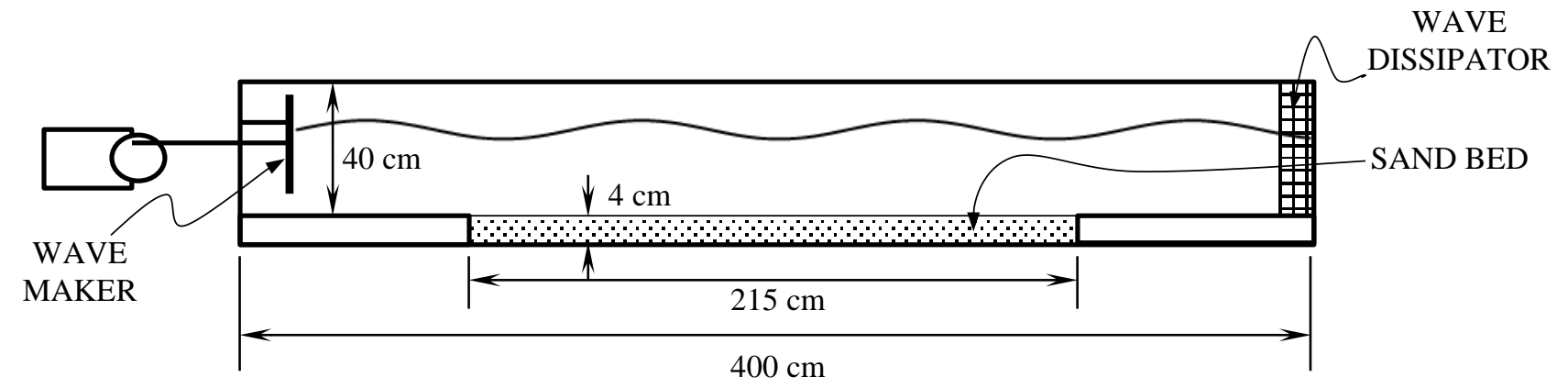

Fig. 1: Sketch of the experimental set-up.

\subsection{Materials}

Two different sizes of sand were used in the bed, with mean diameter $D_{S}$ of 0.25 and $0.55 \mathrm{~mm}$, with $\rho_{S}$ equal to 2600 $\mathrm{kg} / \mathrm{m} 3$. The fluids used in the experiments were water and three pseudoplastic fluids that were produced dissolving sodium carboxymethyl cellulose (CMC) in water, whose rheology is well described by a power law model, $\tau=K \dot{\gamma}^{n}$, where $\tau$ is the shear stress, $\dot{\gamma}$ is the shear rate, $K$ is the consistency coefficient and $n$ is the flow behaviour index. Rheology was determined with a rheometer made by Anton Paar model Rheolab QC, with a peltier temperature control. A summary of the values of $K$ and $n$ is given in Table 1 .

Table 1: Rheological characterization of the pseudoplastic fluids.

\begin{tabular}{|c|c|c|}
\hline FLUID I.D. & $\begin{array}{c}K \\
\mathrm{~Pa} \cdot \mathrm{s}^{\mathrm{n}}\end{array}$ & $n$ \\
\hline PL1 & 0.095 & 0.819 \\
\hline PL2 & 0.081 & 0.822 \\
\hline PL3 & 0.229 & 0.734 \\
\hline
\end{tabular}

\subsection{Wave characterization}

To characterize the waves, the free surface was recorded during the experiments by means of a camera located perpendicular to one of the walls. From the video analysis, the period $T$, wave height $H$, and wave length $L$ were determined. The range covered by these variables in the experiments, and the mean water depth, $h$, is presented in Table 2.

Table 2: Range of variables in the experiments.

\begin{tabular}{|c|c|c|c|}
\hline $\begin{array}{c}T \\
\mathrm{~s}\end{array}$ & $\begin{array}{c}H \\
\mathrm{~cm}\end{array}$ & $\begin{array}{c}L \\
\mathrm{~cm}\end{array}$ & $\begin{array}{c}h \\
\mathrm{~cm}\end{array}$ \\
\hline $0.9-1.9$ & $1.9-6.4$ & $28-110$ & $10-29$ \\
\hline
\end{tabular}

\subsection{Flow regime}

The flow regime was determined using Kamphuis' criterion [11], complemented with visual observation of the flow. Kamphuis' criterion is defined upon a Reynolds number defined as $R e=U_{m} A / v$, where $U_{m}$ and $A$ are the maximum orbital velocity and wave orbital amplitude, just outside boundary layer, respectively. $v$ is the kinematic viscosity of the fluid. In the case of power law fluids, and effective or equivalent viscosity, $v_{e f f}$, was used, defined as $v_{e f f}=\mu_{e f f} / \rho$, where $\mu_{e f f}=K \dot{\gamma}^{n-1}$ and $\rho$ is the fluid density. According to Kamphuis, the upper limit of the laminar regime occurs at $R e \approx 10^{4}$. Excepting one experimental condition with $R e=10022$ (water as fluid), the rest was in the range from 7 to 6931. Thus, all the experiments were in the laminar regime. 


\subsection{Flow characteristics near the bottom}

The wave regime corresponds to the intermediate between deep and shallow water $(0.05<h / L<0.5)$. As a first approximation, the maximum velocity and the semi-excursion of the fluid near the bottom was computed from the Airy's wave theory [12]:

$$
\begin{gathered}
U_{m}=\frac{\pi H}{T \sinh \left(\frac{2 \pi h}{L}\right)} \\
A=\frac{U_{m} T}{2 \pi}=\frac{H}{2 \sinh \left(\frac{2 \pi h}{L}\right)}
\end{gathered}
$$

Although Eqs. 1 and 2 are not valid for viscous fluids, they were used because they can be easily computed from the experimental conditions, and they proved to be good parametrizations in the data analysis.

\subsection{Criterion to determine the threshold condition}

It is well known that the determination of the condition of incipient motion of the particles is rather arbitrary and the origin of the dispersion of the data reported by different researchers [13]. In this research, the threshold condition was defined when a few particles of the bed surface were rolling back and forth.

\section{Scale analysis of the problem}

Let's consider an isolated particle on the bed, which is assumed horizontal, where a laminar flow develops, as shown in Fig. 2. The oscillating flow exerts a drag force, $F_{D}$, on the particle, which is resisted by a Coulombic friction between the particle and the bed. In this analysis the added mass force will be considered negligible when compared with the drag.

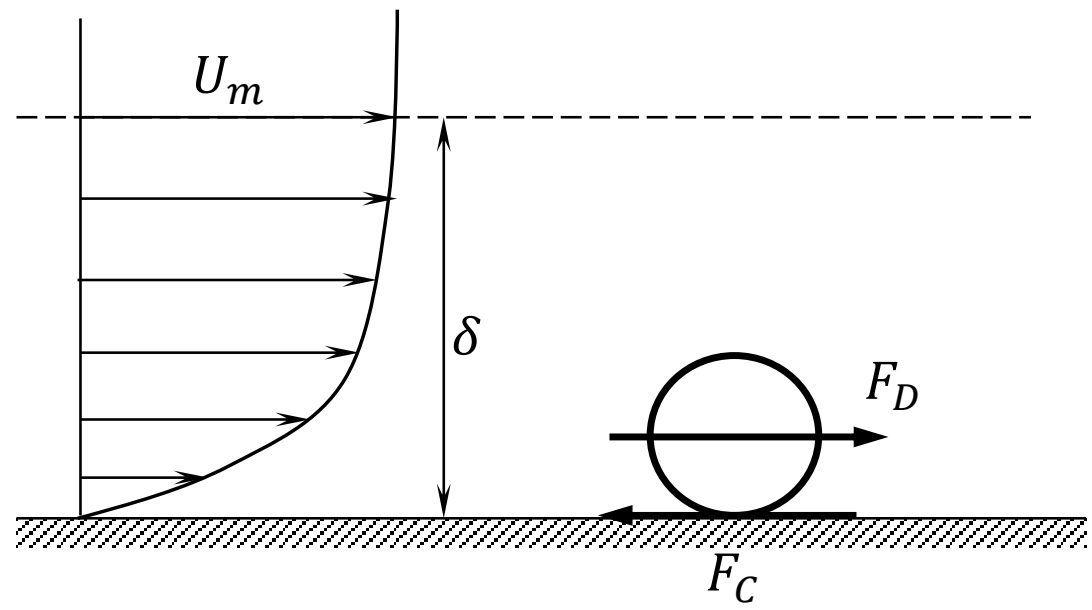

Fig. 2: Forces acting on an isolated particle.

The drag force scales as $F_{D} \sim \rho C_{D} D_{S}^{2} V^{2}$ where $C_{D}$ is the drag coefficient and $V$ a characteristic velocity of the flow. For low Reynolds numbers, in pseudoplastic flows $C_{D} \sim 1 / R e_{p}$, where $R e_{p}$ is a modified Reynolds number [14]. Thus, $F_{D} \sim \mu_{e f f} D_{S} V$. The Coulombic force is proportional to the submerged weight, $F_{C} \sim\left(\rho_{S}-\rho\right) g D_{S}^{3}$, where $g$ is the acceleration due to gravity. A dimensionless parametedefined as the ratio of Coulombic and drag forces, $\Psi=F_{C} / F_{D}$, is introduced in the analysis: 


$$
\Psi=\frac{\rho_{s}-\rho}{\rho} \frac{g D_{S}^{2}}{v_{e f f} V}
$$

A laminar oscillating boundary layer develops over the sand bottom. The scale of the boundary layer thickness is:

$$
\delta \sim \sqrt{v_{e f f} T}
$$

The only other length relevant near the bottom is the particle diameter, originating the dimensionless parameter $X=D_{S} / \delta$ :

$$
X=\frac{D_{S}}{\sqrt{v_{e f f} T}}
$$

Following Goddet [2], it is proposed that $\Psi=\Psi(X)$. This functional relationship will be used to analyse the data in the next section. As characteristic velocity, the maximum orbital velocity near the bottom, $U_{m}$, is used.

Due to its dependency with the shear rate, the effective viscosity varies in time and space. However, a characteristic value of $\dot{\gamma}$ was used in order to quantify $v_{e f f}$. The shear stress on the bed is given by $\tau_{0}=\left.K(d u / d y)^{n}\right|_{0}$, from where an effective dynamic viscosity near the bottom is defined as $\mu_{e f f 0}=\left.K(d u / d y)^{n-1}\right|_{0}$. It is clear that $\mu_{e f f 0}$ is flow dependent. As a first approximation, the expression obtained for a two dimensional, uniform free surface flow is used, $U_{m}$, instead of the mean velocity $U$ :

$$
\mu_{\text {eff }}=\frac{1}{3}\left(\frac{2 n+1}{n}\right)^{n} K\left(\frac{U_{m}}{h}\right)^{n-1}
$$

In general, the effective viscosity for uniform flows can be written as $\mu_{e f f}=F K(U / d)^{n-1}$, where $d$ is a length associated to de flow depth and $F$ is a function of the flow index $[15,16]$. Thus, for example, in a cylindrical duct $d$ corresponds to the pipe diameter, and $F=((3 n+1) / 4 n)^{n}$. Selection of Eq. 6 is justified by the good agreement of the experimental data to the final relationship that is found for the incipient condition, as it will be seen in the following section.

\section{Data analysis}

The relation between the variables $\Psi$ and $X$ is presented in Fig. 3. It is observed that, although there is a tendency of the data to follow a growing relationship between these variables, there is a considerable scatter of the data and they tend to be grouped according to the fluid rheology and sediment size. Thus, the variables need to be modified in order to correct these effects. First, it was considered that a Reynolds number based on the effective viscosity and the particle size $\left(R e_{p}=\right.$ $U_{m} D_{S} / v_{e f f}$ ) would help to collapse the data, and the variable $X$ was replaced by $X_{R e p}=R e_{p}^{\alpha} X$, where the exponent $\alpha$ should be determined by the best fit to the data. Although the relationship between $\Psi$ and $X_{R e p}$ presented a better collapse than that shown in Fig. 3, it still showed a segregation of the data according to its rheology. Thus a new variable was defined, $X_{n}=e^{\beta n} X_{R e p}=e^{\beta n} R e_{p}^{\alpha} X$, which includes another parameter to be fitted to the data, $\beta$.

As it can be seen in Fig. 4, a good collapse of the data is obtained when the variables $\Psi$ and $X_{n}$ are used. A best fit procedure yielded the values $\alpha=-0.26$ and $\beta=2.75$. The condition for incipient motion in oscillatory flow, including data of Newtonian and non-Newtonian fluids is well represented $\left(R^{2}=0.991191\right)$ by the relation:

$$
\Psi=4.768 X_{n}^{-2.815}
$$


with

$$
X_{n}=e^{2.75 n} R e_{p}^{-0.26} \frac{D_{S}}{\sqrt{v_{e f f} T}}
$$

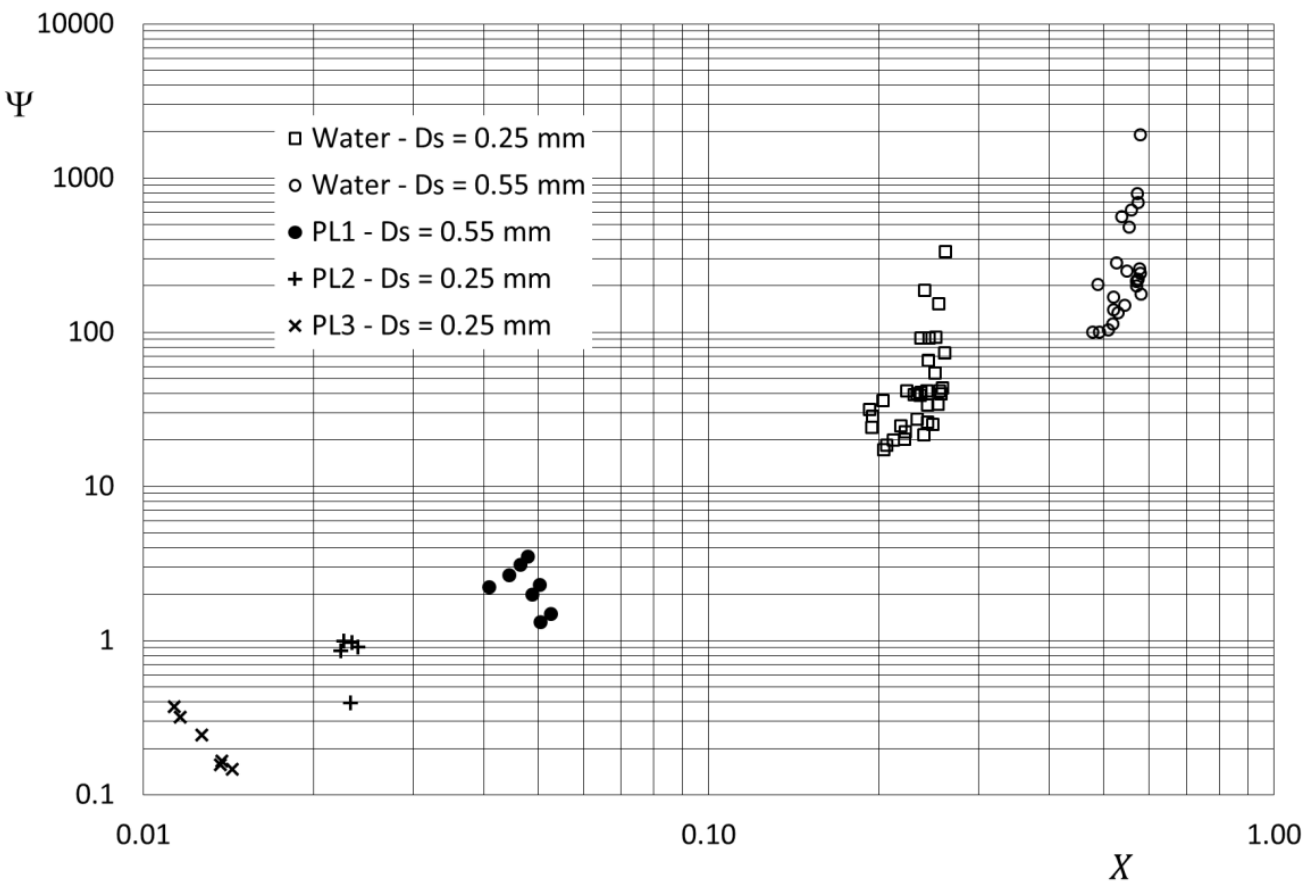

Fig. 3: Condition of incipient motion in terms of the dimensionless variables $\Psi$ and $X$.

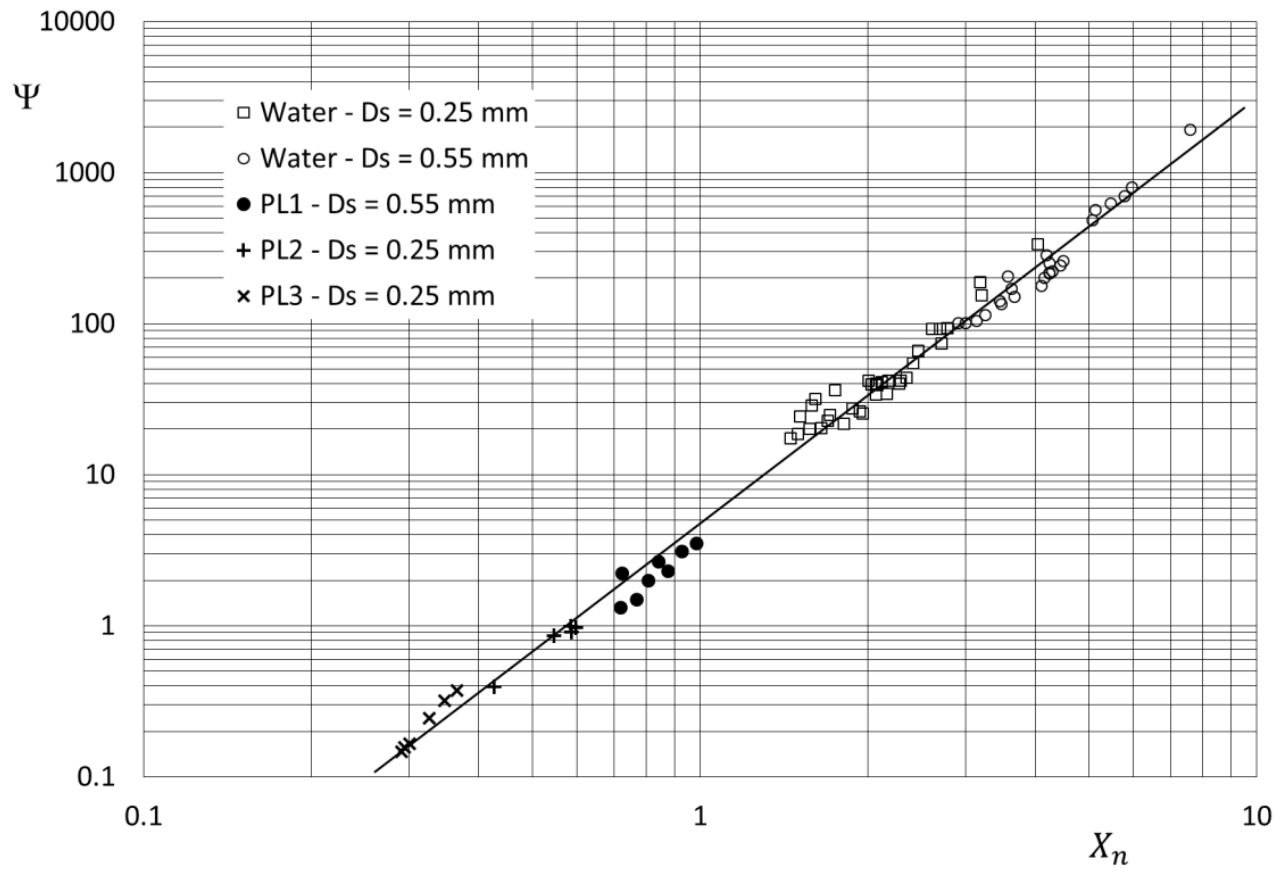

Fig. 4: Condition of incipient motion in terms of the dimensionless variables $\Psi$ and $X_{n}$. The straight line corresponds to Eq. 7 . 


\section{Conclusion}

The experimental data corroborate the choice of the dimensionless variables used in the analysis to determine the condition of incipient motion of granular non-cohesive particles in a laminar oscillating flow of a power-law fluid. The dimensionless variables, $\Psi$ and $X_{n}$, are well correlated and a relationship is proposed to compute the threshold condition. It has to be noted that this relationship includes data obtained with water and pseudoplastic fluids. As it is indicated in the title of the paper, the results are not definitive, as this is a research in development and more data, expanding the spectrum of experimental conditions, are required to generalize the results presented in this paper. In this regard, a more elaborate analysis of the forces involved in the equilibrium of the particle is required, fluids with a wider range of values of the flow behavior index, as well as a broader range of wave periods and amplitudes.

As far as the authors are aware, the condition of incipient motion in oscillatory flows on non-Newtonian fluids has not been determined before, which highlights the contribution of this article to the fluid dynamics community.

\section{Acknowledgements}

The authors acknowledge the funding provides by the Chilean Fund for Science and Technology by means of the grant Fondecyt 1161751.

\section{References}

[1] R. A. Bagnold, "Motion of waves in shallow water: interaction between waves and sand bottom," Proceedings of the Royal Society of London, vol. 187, pp. 1-15, 1946.

[2] J. Goddet, "Étude du début d'entraînement des matériaux mobiles sous l'action de la houle," La Houille Blanche, no. 2, pp. 122-135, 1960.

[3] P. J. Rance and N. F. Warren, "The Threshold of Movement of CoarseMaterial in Oscillatory Flow," Proceedings of the 11th Conference on Coastal Engineering, London, 1968.

[4] P. D. Komar and M. Miller, "The threshold of sediment movement under oscillatory water waters," Journal of Sedimentary Petrology, vol. 43, pp. 1111-1113, 1973.

[5] J. K. Rigler and M. B. Collins, "Initial grain motion under oscillatory flow: a comparison of some threshold criteria," Geo-Marine Letters, vol. 3, pp. 43-48, 1983.

[6] M. Green, "Test of sediment initial motion theories using irregular wave field data," Sedimentology, vol. 46, pp. 427-441, 1999.

[7] Z. J. You, "A simple model of sediment initiation under waves," Coastal Engineering, vol. 41, pp. 399-412, 2000.

[8] Z. J. You and B.S. Yin, "A unified criterion for initiation of sediment motion and inception of sheet flow under water waves". Sedimentology, vol. 53, pp. 1181-1190, 2006.

[9] C.-O. Ng "Mass transport in a layer of power-law fluid forced by periodic surface pressure," Wave Motion, vol. 39, pp. 241-259, 2004.

[10] C.-O. Ng and X. Zhang "Mass transport in water waves over a thin layer of soft viscoelastic mud," J. Fluid Mech., vol. 573, pp. 105-130, 2007.

[11] J.W. Kamphuis, "Friction factor under oscillatory waves," Journal of the Waterways, Harbors and Coastal Engineering Division, ASCE. vol. 101, no. WW2, pp. 135-144. 1975.

[12] US Corp of Engineers, Coastal Engineering Manual - Part II, Publication number EM 1110-2-1100, 2002.

[13] L. Lenhoff, "Incipient motion of particles under oscillatory flow," Proceedings of 18th Conference on Coastal Engineering, Cape Town, South Africa, 1982.

[14] R. P. Chhabra, Bubbles, Drops, and Particles in Non-Newtonian Fluids. CRC Press. Taylor \& Francis Group. Cambridge, 2007.

[15] W. Kozicki, C. H. Chou and C. Tiu, "Non-Newtonian flow in ducts of arbitrary cross-sectional shape," Chemical Engineering Science, vol. 21, pp. 665-679, 1966.

[16] F. Delplace and J. C. Leuliet, "Generalized Reynolds number for the flow of power law fluids in cylindrical ducts of arbitrary cross-section,” The Chemical Engineering Journal, vol. 55, pp. 33-37, 1995. 